\title{
液相沉积法制备 $\mathrm{ZnO} / \mathrm{CdS}$ 复合纳米棒阵列薄膜及其光电性质
}

\author{
付冬伟程轫庞山杜祖亮 * \\ (河南大学特种功能材料教育部重点实验室, 河南开封 475004)
}

\begin{abstract}
摘要: 采用两步化学溶液沉积法在氧化铟锡(ITO) 导电玻璃衬底上制备了 $\mathrm{ZnO} / \mathrm{CdS}$ 复合纳米棒阵列薄膜. 利用 $X$ 射线衍射 $(X R D)$ 仪、扫描电子显微镜 (SEM)、紫外-可见(UV-Vis)吸收分光光度计、苂光(PL)光谱仪及表面光电 压谱(SPS)研究了不同 CdS 沉积时间对复合薄膜的晶体结构、形貌、光电性质的影响. 研究结果表明: $\mathrm{ZnO}$ 纳米棒 阵列表面包覆 CdS 纳米颗粒后, 其吸收光谱可拓展到可见光区; 与吸收光谱相对应在可见光区出现新的光电压 谱响应区, 这一现象证实, 通过与 $\mathrm{CdS}$ 复合可显著提高 $\mathrm{ZnO}$ 纳米棒阵列在可见光区的光电转换性能; 随着 $\mathrm{CdS}$ 纳米颗粒沉积时间的延长, 复合纳米棒阵列薄膜在大于 $383 \mathrm{~nm}$ 波长区域的光电压强度逐渐减弱, 而在小于 383 $\mathrm{nm}$ 波长区域的光电压强度逐渐增强. 用两种不同的电荷产生和分离机制对这一截然相反的光响应过程进行了 详细的讨论和解释.
\end{abstract}

关键词： $\mathrm{ZnO} / \mathrm{CdS}$ 纳米棒阵列; 液相沉积法; 表面光电压谱; 光电特性; 电荷转移过程 中图分类号: $\mathrm{O} 649 ; \mathrm{O} 484$

\section{Solution Based Synthesis of ZnO/CdS Composite Nanorod Array Film and Its Photoelectric Properties}

\author{
FU Dong-Wei CHENG Ke PANG Shan DU Zu-Liang" \\ (Key Laboratory for Special Functional Materials of Ministry of Education, Henan University, Kaifeng 475004, \\ Henan Province, P. R. China)
}

\begin{abstract}
Well-aligned $\mathrm{ZnO} / \mathrm{CdS}$ composite nanorod array film was grown on an indium tin oxide (ITO) substrate by two-step chemical solution deposition method. The effects of CdS deposition time on the crystal structure, morphology, and photoelectric performance of the $\mathrm{ZnO} / \mathrm{CdS}$ composite film were investigated by X-ray diffraction (XRD), scanning electron microscopy (SEM), ultraviolet-visible absorption spectroscopy (UV-Vis), photoluminescence spectroscopy (PL), and surface photovoltage spectroscopy (SPS). Results showed that the absorbance of the composite film extended into the visible region compared with the bare $\mathrm{ZnO}$ nanorod arrays. SPS also showed a new response region corresponding to the absorption spectrum. This result indicated a remarkable photoelectric conversion efficiency improvement in the visible region. We also found that the SPS response intensity of the composite film decreased gradually above $383 \mathrm{~nm}$ with an increase in CdS deposition time. However, the SPS response intensity increased below $383 \mathrm{~nm}$. We interpreted this phenomenon using two distinct photoinduced charge generation and transfer mechanisms.
\end{abstract}

Key Words : $\mathrm{ZnO} / \mathrm{CdS}$ nanorod array; Solution deposition; Surface photovoltage spectrum;

Photo-electric property; Charge transfer process

$\mathrm{ZnO}$ 纳米结构因具有优越的光电子性能已被 作为染料敏化太阳能电池(DSSC)的光阳极材料应
用到纳米结构太阳能电池中 ${ }^{[1-4]}$. 但 $\mathrm{ZnO}$ 是一种宽带 隙半导体材料 $\left(E_{\mathrm{g}} \approx 3.3 \mathrm{eV}\right)$, 几乎不能吸收和利用太

Received: March 24; Revised: May 25, 2010; Published on Web: July 21, 2010.

*Corresponding author. Email: zld@henu.edu.cn; Tel: +86-378-3881358.

The project was supported by the National Natural Science Foundation of China $(20773103,90306010,10874040)$.

国家自然科学基金 $(20773103,90306010,10874040)$ 资助项目

C Editorial office of Acta Physico-Chimica Sinica 
阳光谱中可见区域的光. 为了克服 $\mathrm{ZnO}$ 自身对可见 光利用的局限性, 已有不少小组将其与窄带隙半导 体材料复合来改善 $\mathrm{ZnO}$ 的光吸收性能 ${ }^{[5-6]}$. 而用纳 米粒子做为电池光阳极材料, 通常认为粒间扩散是 纳米颗粒薄膜电子传输的主要机理, 由于粒子之间 存在较多的界面势垒, 电子在其中扩散传输时容易 在纳米粒子之间的界面发生复合, 从而效率不高 ${ }^{[7-9]}$. 因此有的研究小组采用一维 $\mathrm{ZnO}$ 纳米阵列来充当 太阳能电池的阳极材料, 在增大异质结界面的同时, 也为电子传输到相应的电极提供了直接通道, 减小 了在纳米晶颗粒之间扩散过程中由于通过太多的粒 子界面而造成的复合损失 ${ }^{[10-12]}$.

目前一维 $\mathrm{ZnO}$ 纳米阵列结构主要由液相法、气 相法及电沉积等方法制备, 并被广泛作为制备纳米 棒阵列复合薄膜太阳能电池的阳极材料 ${ }^{[10-15]}$. 在这 些方法中液相法因其低温、低成本、可大规模制备且 能制备高密度纳米结构阵列而备受人们青睐与推 崇. 最近, Tak 等 ${ }^{[16]}$ 详细研究了 $\mathrm{CdS}$ 纳米粒子在 $\mathrm{ZnO}$ 纳米阵列上的生长动力学过程; Lee 等 ${ }^{[17]}$ 基于液相 法在 $\mathrm{ZnO}$ 纳米棒阵列上制备了 $\mathrm{CdS}$ 量子点敏化的 太阳能电池. 尽管已经有部分研究小组构筑了 $\mathrm{ZnO} /$ $\mathrm{CdS}$ 复合纳米棒阵列薄膜, 并对其光电转换能力进 行了表征, 但很少有人关注该复合体系中的光生电 荷产生、分离和传输过程, 限制了其进一步的应用. 因此在本文中, 我们基于两步液相化学溶液沉积法 在 ITO 衬底上制备了 $\mathrm{ZnO}$ 纳米棒/ $\mathrm{CdS}$ 纳米粒子复 合阵列薄膜, 考察了不同 $\mathrm{CdS}$ 沉积时间对其结构、 形貌及光电性能的影响, 并利用表面光电压谱对该 复合体系中光生电荷的产生、分离和传输过程进行 了详细的研究, 我们的实验结果对于改善这种复合 体系的电荷分离效率, 发展新型的高性能光电器件 提供了实验和理论基础.

\section{1 实验部分}

\section{1 试剂与仪器}

硝酸锌 $\left(\mathrm{Zn}\left(\mathrm{NO}_{3}\right)_{2} \cdot 6 \mathrm{H}_{2} \mathrm{O}, \geqslant 99.5 \%\right)$ 、乙酸锌 $\left(\left(\mathrm{CH}_{3} \mathrm{COO}\right)_{2} \mathrm{Zn} \cdot 2 \mathrm{H}_{2} \mathrm{O}, \geqslant 99.0 \%\right)$ 、六次甲基四胺 $\left(\mathrm{C}_{6} \mathrm{H}_{12} \mathrm{~N}_{4}, \geqslant 99.0 \%\right)$ 、乙 酸镉 $\left(\left(\mathrm{CH}_{3} \mathrm{COO}\right)_{2} \mathrm{Cd} \cdot 2 \mathrm{H}_{2} \mathrm{O}\right.$, $\geqslant 99.5 \%)$ 、乙酸铵 $\left(\mathrm{CH}_{3} \mathrm{COONH}_{4}, \geqslant 98.0 \%\right)$ 均为分 析纯, 购于天津市科密欧化学试剂有限公司; 硫嫝 $\left(\mathrm{H}_{2} \mathrm{NCSNH}_{2}, \geqslant 99.0 \%\right)$ 为分析纯, 购于天津市精细化 工研究所; 无水乙醇 $\left(\mathrm{CH}_{3} \mathrm{CH}_{2} \mathrm{OH}, \geqslant 99.7 \%\right)$ 为分析 纯, 购于中国医药公司; 氨水 $\left(\mathrm{NH}_{3} \cdot \mathrm{H}_{2} \mathrm{O}, 25 \%-28 \%\right)$
为分析纯, 购于洛阳化学试剂厂; 实验用水为自制三 次蒸馏水.

$X$ 射线衍射仪 $(X R D)$ 采用荷兰 Philips 公司的 $\mathrm{X}^{\prime}$ Pert Pro MPD 型衍射仪 $\left(\mathrm{Cu}\right.$ 靶 $\left.K_{\alpha}\right)$; 扫描电子显微 镜 (SEM) 采用日本电子株式会社 (JEOL Ltd.) 的 JSM5600LV 型扫描电镜; 紫外-可见(UV-Vis)吸收分 光光度计采用英国 UNICAM 公司的 HEXIOS $\alpha$ 型 紫外-可见吸收分光光度计; 苂光(PL)光谱仪采用美 国 SPEX 公司的 SPEX-F212 型苂光谱仪; 表面光电 压谱(SPS)采用美国 Stanford 公司的 SR830 型光电 压谱仪.

\section{2 实验过程}

\subsubsection{ZnO 纳米棒阵列的合成}

基于两步液相化学溶液沉积法在 ITO 衬底上 制备一维 $\mathrm{ZnO} / \mathrm{CdS}$ 复合纳米棒阵列薄膜. $\mathrm{ZnO}$ 纳米 棒阵列的制备采用类似 Greene 等 ${ }^{[18}$ 报道的方法. 首 先, 用乙酸锌 $\left(0.005 \mathrm{~mol} \cdot \mathrm{L}^{-1}\right)$ 的乙醇溶液将洁净的 ITO 衬底润湿后在空气中晾干, 这样重复三次后将包覆 有乙酸锌晶粒的 ITO 衬底在大气气氛下 $350{ }^{\circ} \mathrm{C}$ 退 火 $30 \mathrm{~min}$, 之后再次执行上述步骤, 最终 ITO 被包 覆上一层均匀的 $\mathrm{ZnO}$ 纳米晶种子层. 然后, 将包覆 有 $\mathrm{ZnO}$ 种子层的衬底悬置于硝酸锌 $\left(0.05 \mathrm{~mol} \cdot \mathrm{L}^{-1}\right)$ 和六次甲基四胺 $\left(0.05 \mathrm{~mol} \cdot \mathrm{L}^{-1}\right)$ 的水溶液中 $95{ }^{\circ} \mathrm{C}$ 生 长 $3 \mathrm{~h}$, 取出并用水完全冲洗获得 $\mathrm{ZnO}$ 纳米棒阵列, 最后, 将 $\mathrm{ZnO}$ 阵列在空气中 $350{ }^{\circ} \mathrm{C}$ 煅烧 $30 \mathrm{~min}$ 除掉 残留物以备下一步沉积 $\mathrm{CdS}$ 纳米粒子用.

\subsection{2 $\mathrm{ZnO} / \mathrm{CdS}$ 复合纳米棒阵列的制备}

将生长有 $\mathrm{ZnO}$ 纳米棒阵列的衬底悬置于 100 $\mathrm{mL}$ 的乙酸镉 $\left(0.001 \mathrm{~mol} \cdot \mathrm{L}^{-1}\right)$ 、硫腺 $\left(0.005 \mathrm{~mol} \cdot \mathrm{L}^{-1}\right)$ 、 乙酸铵 $\left(0.006 \mathrm{~mol} \cdot \mathrm{L}^{-1}\right)$ 和氨水 $\left(0.4 \mathrm{~mol} \cdot \mathrm{L}^{-1}\right)$ 混合的 70 ${ }^{\circ} \mathrm{C}$ 水溶液中, 并慢速磁力搅拌沉积 $\mathrm{CdS}$, 之后将样品 取出并立即用水冲洗掉残液, 干燥后获得 $\mathrm{ZnO} / \mathrm{CdS}$ 复合纳米棒阵列薄膜.

\section{2 结果与讨论}

\section{$2.1 \mathrm{ZnO} / \mathrm{CdS}$ 复合纳米棒阵列薄膜的结构与形貌}

图 1 为 $\mathrm{ZnO}$ 纳米棒阵列和不同 $\mathrm{CdS}$ 沉积时间 后的 $\mathrm{ZnO} / \mathrm{CdS}$ 复合结构的 XRD 图谱. 从图中可以 看出, 沉积 $\mathrm{CdS}$ 之前 $\mathrm{ZnO}$ 纳米棒阵列的 $(002)$ 晶面 峰强度远大于其他各晶面峰强度, 表明 $\mathrm{ZnO}$ 纳米棒 阵列更倾向于沿垂直衬底的 $c$ 轴取向生长 ${ }^{[19]}$. 沉积 $\mathrm{CdS}$ 之后, $\mathrm{ZnO}$ 阵列的特征衍射峰仍然存在, 说明在 $\mathrm{CdS}$ 沉积过程中, $\mathrm{ZnO}$ 纳米棒阵列结构并没有遭到 


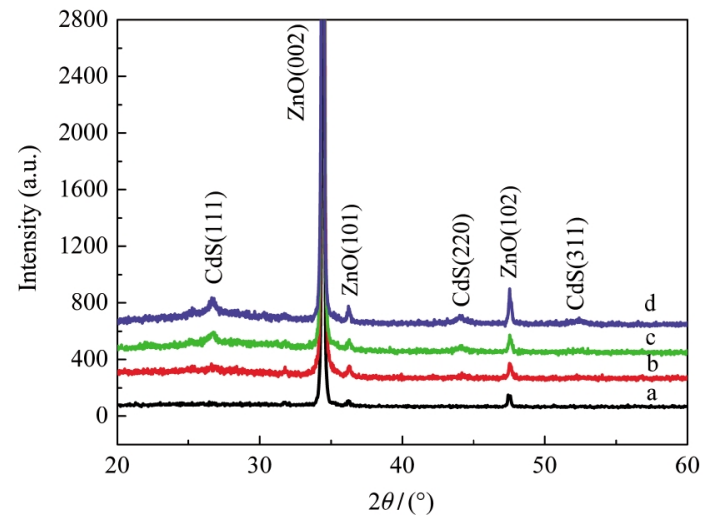

图 $170{ }^{\circ} \mathrm{C}$ 沉积不同时间 $\mathrm{ZnO} / \mathrm{CdS}$ 复合纳米棒阵列的 XRD 图

Fig.1 XRD patterns of $\mathrm{ZnO} / \mathrm{CdS}$ composite nanorod arrays deposited at $70{ }^{\circ} \mathrm{C}$ for different time t/min: (a) 0; (b) 5; (c) 10; (d) 20

强碱性溶液 $(\mathrm{pH} \approx 11)$ 的破坏. 另一方面, 随着 $\mathrm{CdS}$ 沉积时间的延长, $\mathrm{CdS}$ 衍射峰强度也逐渐增强, 但是 衍射峰宽化程度逐渐减弱, 说明随着 $\mathrm{CdS}$ 沉积时间 的增长, 其晶粒尺寸也在逐渐变大 ${ }^{[20]}$.

对 $\mathrm{ZnO}$ 纳米棒阵列与 $\mathrm{CdS}$ 复合前后的形貌也 做了 SEM 表征, 结果如图 2 所示. 由图 2(a)俯视图 和图 2(e)截面图可知, $\mathrm{ZnO}$ 纳米棒的平均直径约为 $160 \mathrm{~nm}$, 长度约为 $1.9 \mu \mathrm{m}$, 并且它们都几乎垂直于 衬底进行生长, 与从 XRD 看到的具有取向生长相 一致. 图 2(b-d) 分别为 $\mathrm{CdS}$ 沉积时间为 $5 、 10$ 和 20 $\min$ 之后的形貌, 与沉积前相比, $\mathrm{ZnO}$ 纳米棒阵列结
构并没有受到碱性溶液 $(\mathrm{pH} \approx 11)$ 的显著破坏, 这 也与 XRD 结果相一致; 由 SEM 图可以清晰地看出, 随着 $\mathrm{CdS}$ 沉积时间的延长, $\mathrm{ZnO}$ 纳米棒的直径在逐 渐增加, 棒与棒的间隙随沉积时间的延长逐渐减小, 沉积 $20 \mathrm{~min}$ 时有些纳米棒之间的间隙已完全被 $\mathrm{CdS}$ 填充. 从图 2(e,f)的截面图已可清晰看出, 沉积 前 $\mathrm{ZnO}$ 纳米棒比较光滑, 而当沉积 $\mathrm{CdS} 5 \mathrm{~min}$ 后, $\mathrm{ZnO}$ 纳米棒的表面已变得十分粗糙, 可以清楚地看 出其表面被一层 $\mathrm{CdS}$ 纳米粒子所覆盖, 我们的 $\mathrm{XRD}$ 结果也证实, 经过 $\mathrm{CdS}$ 沉积后, 存在 $\mathrm{CdS}$ 的衍 射峰, 这说明采用这种水浴沉积的方法可以较好地 将 $\mathrm{CdS}$ 纳米粒子和 $\mathrm{ZnO}$ 纳米棒阵列进行复合.

\section{2 光学性质}

由于 $\mathrm{CdS}$ 是窄带隙半导体 $\left(E_{\mathrm{g}} \approx 2.4 \mathrm{eV}\right)$, 而 $\mathrm{ZnO}$ 的带隙要比 $\mathrm{CdS}$ 的宽, 这样通过 $\mathrm{ZnO}$ 与 $\mathrm{CdS}$ 复合可 以改变 $\mathrm{ZnO}$ 的光吸收特性. 图 3 给出了 $\mathrm{ZnO}$ 纳米 棒阵列在沉积 $\mathrm{CdS}$ 纳米颗粒前后的 UV-Vis 吸收 谱, 由图中可以看出纯 $\mathrm{ZnO}$ 在可见光区吸收较弱, 当它与 $\mathrm{CdS}$ 复合后其可见区的光吸收性能得到明 显提高, 并且吸收强度随着 $\mathrm{CdS}$ 沉积时间的延长而 逐渐增强. $\mathrm{ZnO}$ 纳米棒阵列上沉积 $\mathrm{CdS} 5 \mathrm{~min}$ 后其 吸收边已由沉积前近紫外区拓展到了可见光区域 $580 \mathrm{~nm}$ 附近, 而且随着 CdS 沉积时间的延长, 最终 $\mathrm{ZnO} / \mathrm{CdS}$ 复合结构的吸收边也逐渐红移至 $600 \mathrm{~nm}$ 处, 发生红移的原因应归因于沉积过程中 $\mathrm{CdS}$ 晶粒 尺寸的逐渐增大所导致, 这与 XRD 结果的 $\mathrm{CdS}$ 衍

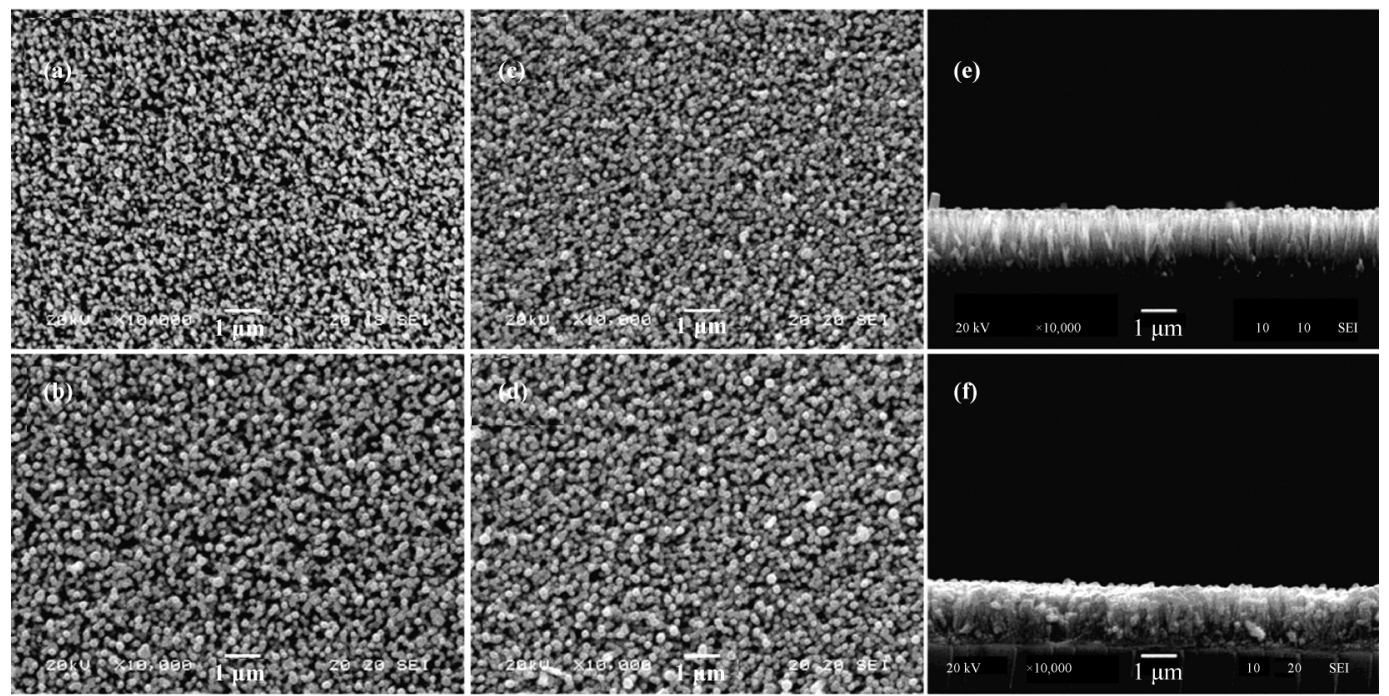

图 2 不同 $\mathrm{CdS}$ 沉积时间所得 $\mathrm{ZnO} / \mathrm{CdS}$ 复合纳米棒阵列的平面和截面 SEM 照片

Fig.2 Plan-view and the cross-section SEM images of $\mathrm{ZnO} / \mathrm{CdS}$ composite nanorod arrays obtained at different CdS deposition time

$t / \min :(\mathrm{a}, \mathrm{e}) 0$; (b, f) 5 ; (c) 10 ; (d) 20 
射峰半峰宽随沉积时间增加而逐渐减小结果相一 致, 同样的实验现象也有类似报道 ${ }^{[2]}$.

为了进一步了解其光学性质, 我们对样品也做 了荧光(PL)测试. 从图 4 明显可以看出, 复合前 $\mathrm{ZnO}$ 纳米棒阵列存在两个发光带, 一个发光中心峰位于 $386 \mathrm{~nm}$ 附近的紫外区, 另一个位于 $595 \mathrm{~nm}$ 附近的 范围较宽的可见光区, 紫外区发光主要源自光生载 流子近带边跃迁复合, 而较宽的可见区发光通常认 为是由于 $\mathrm{ZnO}$ 中存在氧离子空位所导致的表面态 发光 ${ }^{[22-23]}$. 在沉积 $\mathrm{CdS}$ 后, 紫外区的发光中心向短波 段移动了约 $4 \mathrm{~nm}$, 发光峰蓝移通常认为是由于溶液 中的硫阴离子被 $\mathrm{ZnO}$ 表面的氧空位俘获, 然后进一 步向 $\mathrm{ZnO}$ 纳米棒内部扩散并作为施主杂质而存在, 这样硫掺杂原子提供的过量载流子就会填充 $\mathrm{ZnO}$ 的导带底, 从而导致光学带-带跃迁蓝移的产生, 这 种现象在硫和镁掺杂的 $\mathrm{ZnO}$ 中都已被观察到 ${ }^{[24-25]}$. 此外, 硫阴离子在 $\mathrm{ZnO}$ 纳米棒表面的俘获同时为 $\mathrm{CdS}$ 的沉积提供了最初成核位 ${ }^{[16]}$.

\section{3 光电特性表征}

表面光电压谱(SPS)技术是研究表界面光电分 离与传输过程的有效工具 ${ }^{[26-29]}$, 这里用 SPS 对 $\mathrm{ZnO} /$ $\mathrm{CdS}$ 复合结构进行了表征, 结果见图 5. 从图中可以 观察到, 单纯的 $\mathrm{ZnO}$ 纳米棒阵列的光电压强度较 弱, 只在 $383 \mathrm{~nm}$ 附近出现了一个光电压的响应峰, 这是由于 $\mathrm{ZnO}$ 纳米棒阵列受到光激发而产生的价 带-导带电荷跃迁分离所导致 ${ }^{[30]}$, 从吸收光谱可以看 出, 纯的 $\mathrm{ZnO}$ 纳米棒阵列在可见光区没有光吸收, 与此相对应, 在表面光电压谱表征中, 我们也没有观

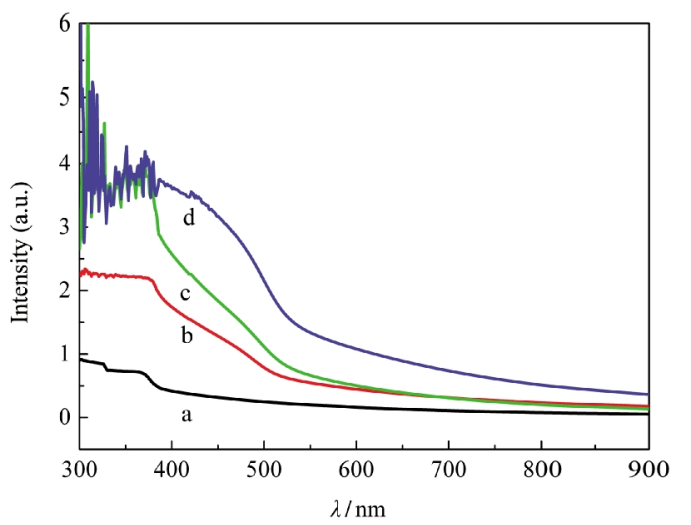

图 3 不同 CdS 沉积时间所得 $\mathrm{ZnO} / \mathrm{CdS}$ 复合陈列薄膜的 紫外-可见吸收光谱

Fig.3 UV-Vis absorbance spectra of $\mathrm{ZnO} / \mathrm{CdS}$ composite nanorod array film obtained at different CdS deposition time t/min: (a) 0; (b) 5; (c) 10; (d) 20

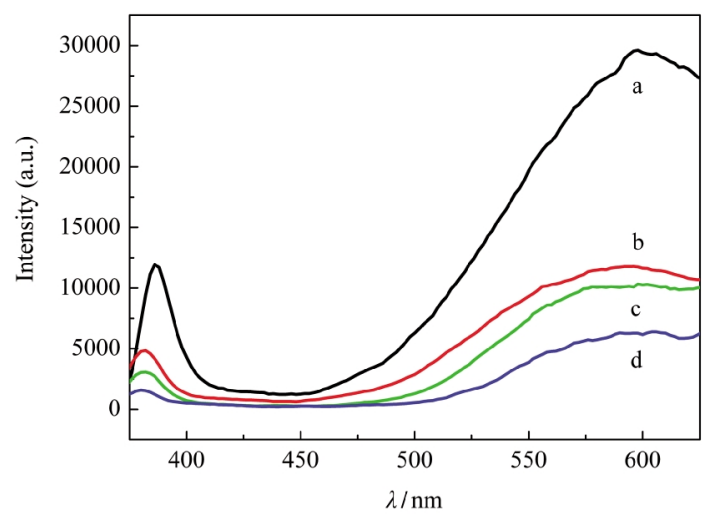

图 4 不同 $\mathrm{CdS}$ 沉积时间所得 $\mathrm{ZnO} / \mathrm{CdS}$ 复合陈列薄膜的 光致发光谱图

Fig.4 Photoluminescence spectra of $\mathrm{ZnO} / \mathrm{CdS}$ composite nanorod array film obtained at different CdS deposition time t/min: (a) 0 ; (b) 5 ; (c) 10 ; (d) 20

察到在可见光区的光电响应. 当 $\mathrm{ZnO}$ 纳米棒阵列和 $\mathrm{CdS}$ 复合后, 光电压强度明显得到增强, 光电响应由 原来的近紫外区扩展到了可见光区, 并且随着 $\mathrm{CdS}$ 沉积时间的延长, 其光电响应也逐步向长波方向扩 展, 这也与 UV-Vis 吸收结果相吻合. 有趣的是我们 观察到两者复合之后, 在大于 $383 \mathrm{~nm}$ 的长波区域, 随着 $\mathrm{CdS}$ 沉积时间的延长, 其光电压强度却逐渐减 弱; 而在短波区域却随着 $\mathrm{CdS}$ 沉积时间的延长, 其 光电压强度逐渐增强. 显然这对应两个完全不同的 光生载流子的产生、分离与传输过程.

图 6(a)展示了样品测试结构示意图, 对上电极 ITO 、样品和衬底 ITO 形成三明治结构进行了 SPS 测试. 由表面光电压谱理论得知, 当激发光波长大于

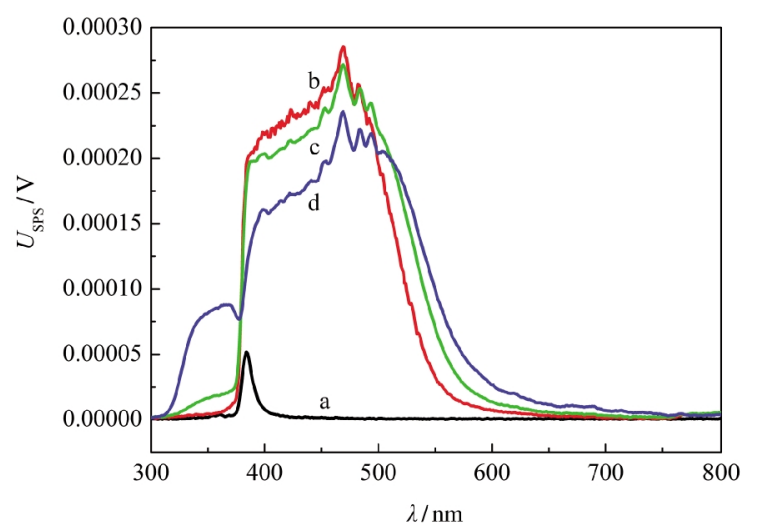

图 5 不同 $\mathrm{CdS}$ 沉积时间所得 $\mathrm{ZnO} / \mathrm{CdS}$ 复合阵列薄膜的 表面光电压谱

Fig.5 Surface photovoltage spectra (SPS) of $\mathrm{ZnO}$ / CdS composite nanorod array film obtained at different CdS deposition time t/min: (a) 0 ; (b) 5 ; (c) 10 ; (d) 20 
(a)

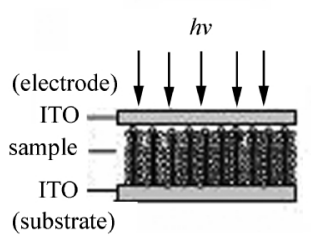

(c)

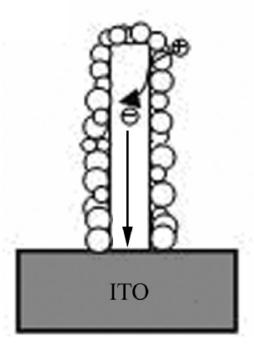

(b)
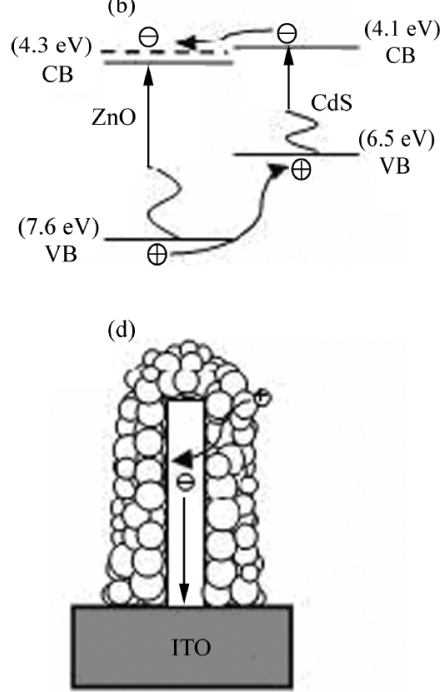

图 $6 \mathrm{ZnO} / \mathrm{CdS}$ 复合薄膜的光电压测试及光诱导电荷传输 示意图

Fig.6 Schematic diagrams of SPS measurement and photo-induced charge transfer process of $\mathrm{ZnO} / \mathrm{CdS}$ composite film

(a) SPS measurement sketch; (b) energy band diagram and photoinduced electron-hole pair transfer; (c, d) morphology evolution of $\mathrm{ZnO} / \mathrm{CdS}$ composite nanorod depending on deposition time; $\mathrm{CB}$ : conduction band, VB: valence band

$\mathrm{CdS}$ 的带隙而小于 $\mathrm{ZnO}$ 的带隙时, 只有 $\mathrm{CdS}$ 可以受 到光激发而产生光生电子-空穴对, 并且由于 $\mathrm{ZnO}$ 的导带低于 $\mathrm{CdS}$ 的导带位置而形成交叉型能带排 布, 如图 6(b)所示 ${ }^{[n]}$, 这种结构可以使 CdS 导带上的 光生电子注人到 $\mathrm{ZnO}$ 的导带, 并沿 $\mathrm{ZnO}$ 纳米棒进 行传输, 实现电子-空穴对的有效分离而产生表面 光电压信号. 并且由 SEM 结果可知, 在沉积 CdS 5 $\min$ 时, $\mathrm{ZnO}$ 纳米棒表面包覆的 $\mathrm{CdS}$ 纳米粒子较薄, 这时 CdS 纳米粒子内的光生电子就很容易注人到 $\mathrm{ZnO}$ 纳米棒中进行传输, 从而实现电子-空穴对很 好的分离, 进而产生较强的表面光电压信号, 电荷传 输示意图如图 6(c) 所示. 然而, 随着沉积时间的延 长, $\mathrm{CdS}$ 纳米粒子的沉积量大大增加如图 6(d) 所示, 这样表面的 CdS 受到激发而产生的电子-空穴对要 传输到 $\mathrm{ZnO}$ 纳米棒阵列上需要多次通过 $\mathrm{CdS}$ 纳米 颗粒的界面, 由于界面势垒的存在导致部分光生电 子-空穴对在 $\mathrm{CdS}$ 纳米颗粒的界面处发生复合 ${ }^{[8-9]}$, 从而使传输到 $\mathrm{ZnO}$ 纳米棒中的净电荷数量减少而 最终导致光电压强度减小 ${ }^{[29]}$. 这样我们就很好地解 释了在长波区域随着 $\mathrm{CdS}$ 沉积时间的延长而表面 光电压谱信号减弱的原因.

而在波长小于 $383 \mathrm{~nm}$ 的短波一侧, 我们观察

到光电压变化却随 $\mathrm{CdS}$ 沉积时间延长而增强, 与长 波区域的变化趋势截然不同. 我们认为这是由于在 短波区域 $\mathrm{ZnO}$ 与 $\mathrm{CdS}$ 两者都可以被激发而发生电 荷跃迁, 当 CdS 沉积量相对较少时, 激发光很容易 就可以透过 $\mathrm{CdS}$ 沉积层使 $\mathrm{ZnO}$ 纳米棒自身受到激 发而发生电荷跃迁, 此时 $\mathrm{ZnO}$ 自身的部分导带能级 被占据, 导致 CdS 中产生的部分电子不能跃迁到 $\mathrm{ZnO}$ 的导带而发生分离后传输到 ITO 电极, 因此其 光电压信号较弱; 而随着 $\mathrm{CdS}$ 沉积量增多, 激发光 很难穿过 $\mathrm{CdS}$ 覆盖层使 $\mathrm{ZnO}$ 得到激发, 此时 $\mathrm{CdS}$ 内的光生电子则可以比较容易地注人到 $\mathrm{ZnO}$ 纳米 棒而发生光生电子-空穴对的有效分离, 导致其在 短波区域的光电压信号强度的增强.

\section{3 结 论}

利用两步液相化学溶液沉积法在 ITO 衬底上 制备了 $\mathrm{ZnO} / \mathrm{CdS}$ 复合纳米棒阵列薄膜, 并对不同沉 积时间的复合薄膜进行了光电性质研究. 结果表明, 在 $\mathrm{CdS}$ 沉积 $5 \mathrm{~min}$ 时, $\mathrm{ZnO}$ 纳米棒表面已被一层 $\mathrm{CdS}$ 纳米粒子所包覆. 通过与 $\mathrm{CdS}$ 的复合, $\mathrm{ZnO}$ 的 光响应拓展到了可见光区. 其光电响应也得到了增 强和扩展, 这说明两者之间发生了界面电荷转移, 提 高了光电转换效率. 观察到了在长波区和短波区出 现了两种截然不同的光电响应, 随着 $\mathrm{CdS}$ 纳米颗粒 沉积时间的延长, 复合纳米棒阵列薄膜在大于 383 $\mathrm{nm}$ 波长区域的光电压强度逐渐减弱, 而在小于 383 $\mathrm{nm}$ 波长区域的光电压强度逐渐增强.

\section{References}

1 Otsuka, A.; Funabiki, K.; Sugiyama, N.; Yoshida, T.; Minoura, H.; Matsui, M. Chemistry Letters, 2006, 35(6): 666

2 Zhang, Q. F.; Chou, T. P.; Russo, B.; Jenekhe, S. A.; Cao, G. Z. Angew. Chem. Int. Edit., 2008, 47: 2402

3 Zhang, Y. Z.; Wu, L. H.; Liu, Y. P.; Xie, E. Q. J. Phys. D-Appl. Phys., 2009, 42: 085105

4 Chu, J. B.; Huang, S. M.; Zhang, D. W.; Bian, Z. Q.; Li, X. D.; Sun, Z.; Yin, X. J. Appl. Phys. A, 2009, 95: 849

5 Hotchandani, S.; Kamat, P. V. J. Phys. Chem., 1992, 96: 6834

6 Ganesh, T.; Mane, R. S.; Cai, G.. Chang, J. H.; Han, S. H. J. Phys. Chem. C, 2009, 113: 7666

7 Dloczik, L.; Ileperuma, O.; Lauermann, I.; Peter, L. M.; Ponomarev, E. A.; Redmond, G.; Shaw, N. J.; Uhlendorf, I. J. Phys. Chem. B, 1997, 101: 10281

8 Duzhko, V.; Timoshenko, V. Y.; Koch, F.; Dittrich, T. Phys. Rev. B, 2001, 64: 075204

9 Pang, S.; Xie, T. F.; Zhang, Y.; Wei, X.; Yang, M.; Wang, D. J.; 
Du, Z. L. J. Phys. Chem. C, 2007, 111: 18417

10 Law, M.; Greene, L. E.; Johnson, J. C.; Saykally, R.; Yang, P. Nature Mater., 2005, 4: 455

11 Leschkies, K. S.; Divakar, R.; Basu, J.; Pommer, E. E.; Boercker, J. E.; Carter, C. B.; Kortshagen, U. R.; Norris, D. J.; Aydil, E. S. Nano Lett., 2007, 7(6): 1793

12 Cao, X.; Chen, P.; Guo, Y. J. Phys. Chem. C, 2008, 112: 20560

13 Tang, Y.; Hu, X.; Chen, M.; Luo, L.; Li, B.; Zhang, L. Electrochimica Acta, 2009, 54: 2742

14 Pasquier, A. D.; Chen, H.; Lu, Y. Appl. Phys. Lett., 2006, 89: 253513

15 Zaera, R. T.; Katty, A.; Bastide, S.; Clément, C. L. Chem. Mater., 2007, 19: 1626

16 Tak, Y.; Hong, S. J.; Lee, J. S.; Yong, K. Crystal Growth \& Design, 2009, 9(6): 2627

17 Lee, W.; Min, S. K.; Dhas. V.; Ogale, S. B.; Han, S. H. Electrochemistry Communications, 2009, 11: 103

18 Greene, L. E.; Law, M.; Tan, D. H.; Montano, M.; Goldberger, J.; Somorjai, G.; Yang, P. Nano Lett., 2005, 5(7): 1231

19 Cheng, K.; Cheng, G.; Wang, S.; Li, L.; Dai, S.; Zhang, X.; Zou, B.; Du, Z. New Journal of Physics, 2007, 9: 214

20 Xu, D.; Gao, A. M.; Deng, W. L. Acta Phys. -Chim. Sin., 2008, 24
(7): 1219 [许 迪, 高爱梅, 邓文礼. 物理化学学报, 2008, 24

(7): 1219]

21 Chang, C. H.; Lee, Y. L. Appl. Phys. Lett., 2007, 91: 053503

22 Meng, X. Q.; Zhao, D. X.; Zhang, J. Y.; Shen, D. Z.; Lu, Y. M.; Fan, X. W.; Wang, X. H. Materials Letters, 2007, 61: 3535

23 Han, D.; Zhang, S. C. Acta Phys. -Chim. Sin., 2008, 24(3): 539 [韩 冬, 张树朝. 物理化学学报, 2008, 24(3): 539]

24 Geng, B. Y.; Wang, G. Z.; Jiang, Z.; Xie, T.; Sun, S. H.; Meng, G. W.; Zhang, D. L. Appl. Phys. Lett., 2003, 82(26): 4791

25 Wei, Q.; Li, M. K.; Yang, Z.; Cao, L.; Zhang, W.; Liang, H. W. Acta Phys. -Chim. Sin., 2008, 24(5): 793 [魏 强, 李梦辑, 杨 志, 曹 璐, 张 威, 梁红伟. 物理化学学报, 2008, 24(5): 793]

26 Kronik, L.; Shapira, Y. Surface Science Reports, 1999, 37: 1

27 de Souza, C. F.; Ruda, H. E.; Fafard, S. Journal of Electroanalytical Chemistry, 2003, 559: 49

28 Cheng, K.; He, Y. P.; Miao, Y. M.; Zou, B. S.; Wang, Y. G.; Wang, T. H.; Zhang, X. T.; Du, Z. L. J. Phys. Chem. B, 2006, 110: 7259

29 Ji, Y. L.; Cheng, K.; Zhang, H. M.; Zhang, X. T.; Li, Y. C.; Du, Z. L. Chinese Science Bulletin, 2008, 53(1): 46

30 Belaidi, A.; Dittrich, T.; Kieven, D.; Tornow, J.; Schwarzburg, K.; Kunst, M.; Allsop, N.; Lux-Steiner, M. C.; Gavrilov, S. Solar Energy Materials \& Solar Cells, 2009, 93: 1033 